\section{Determinantes de la competitividad de un destino turístico: caso Villa de Leyva'}

Determinants of competitiveness of a tourist destination: case Villa de Leyva

\author{
Eduardo Antonio Casas Ochoa*
}

Recibido: 11 de octubre de 2014 / Revisado: 12 de diciembre de 2014

Aceptado: 4 de mayo de 2015

\section{Resumen}

Ante la importancia del turismo como actividad estratégica, este se ha incluido en las diversas agendas de competitividad regional como una de las apuestas productivas, en las que se vislumbra a Boyacá como destino turístico de clase mundial. Por eso en esta investigación se analizan las condiciones de competitividad de Villa de Leyva como destino turístico nacional e internacional, mediante la aplicación del modelo de Porter (1991), conocido como "diamante dinámico", que integra cuatro grandes factores, treinta variables, mediante la técnica Delphi.

Palabras clave: destinos turísticos, competitividad, enfoque macroeconómico, ventajas absolutas, ventajas comparativas, ventajas competitivas.

1 Artículo de Investigación

* Economista de la UPTC, especialista en Administración Pública de la ESAP, Mg Economía de la AAU, profesor asociado de la Facultad de Ciencias Administrativas y Contables Universidad de Boyacá. Correo electrónico: eacasas@uniboyaca.edu.co

\section{Abstract}

Given the importance of tourism as a strategic activity, it has been included in the various agendas of regional competitiveness as one of the production positions, in which Boyacá can be seen as a world-class tourist destination. Therefore this research analyzes the conditions competitiveness of Villa de Leyva as national and international tourist destination, by applying the model of Porter (1991), known as "dynamic diamond", which integrates four major factors, 30 variables, using the Delphi technique.

Keywords: tourist destinations, competitiveness, macroeconomic approach, absolute advantages, comparative advantages, compet tive advantages. 


\section{Citación}

Casas, E. (2015). Determinantes de la competitividad de un destino turístico:

\section{Introducción}

Indudablemente, el turismo como sector estratégico ha llegado a convertirse en la principal actividad económica del mundo, con gran valor agregado, compromiso gubernamental y aporte del sector privado, que se muestra promisorio para el desarrollo de las comunidades receptoras si adoptan elementos atractivos, creativos, "mágicos" e integradores como la hospitalidad, la asociatividad y la sostenibilidad, entre otros.

El Gobierno colombiano en sus diversos niveles es consciente de estas premisas y ha incluido al turismo como un sector estratégico. Por eso, cuando se ha pensado que el turismo es una de las apuestas productivas de una región como Boyacá, con sus potencialidades de desarrollo, es porque se ha considerado que la competitividad puede permitir a las empresas sobreponerse a las limitaciones de sus reducidos mercados internos para incursionar con éxito en los mercados internacionales. En ese orden de ideas, el municipio de Villa de Leyva, con gran vocación turística por sus atractivos naturales, culturales e históricos y una economía rural con un pequeño centro histórico, se ha convertido en uno de los referentes de la industria turística del país, con registro de visitantes internacionales.

Sin embargo, para Villa de Leyva, por falta de formación, instrumentos y mecanismos para adaptarse a las nuevas necesidades y realidades que demanda la actividad turística, no se conoce el nivel de competitividad ni sus determinantes, pues los diversos actores del sistema turismo han implementado estrategias para mejorar, sin que generen ni promuevan un sentido de apropiación, de transmisión y cohesión social en el nivel local, ni han logrado los efectos esperados. De lo anterior surge la necesidad de estudiar los determinantes de la competitividad de un destino turístico, de aproximarse a la comprensión de lo que significa la competitividad. Por ello es necesario aclarar la ubicación teórica para acercarse a la temática adoptada en el presente artículo. 


\section{Referente conceptual sobre la competitividad de los destinos turísticos}

En la actual economía de mercado, cada uno de los destinos turísticos debe enfrentar una fuerte competencia $y$, a su vez, cada destino tiene unos factores competitivos que es necesario estudiar. Pero: ¿Qué es la competitividad? Para Ivanchevich, Lorenzi, Skinner y Crosby (1996), es la medida en que una nación, bajo condiciones de mercado libre y leal es capaz de producir bienes y servicios que puedan superar con éxito la prueba de los mercados internacionales, manteniendo y aun aumentando al mismo tiempo la renta real de sus ciudadanos.

Ahora, por analogía, el principio de la ventaja absoluta de Adam Smith (1776)², aplicada a productos tangibles, se aplica a los destinos turísticos que tienen factores que determinan su eficiencia y se sustenta en el menor costo de producción del servicio con respecto a los costos en otros países.

Sin embargo, las condiciones de competitividad de un destino van más allá de tener ventajas absolutas. Según Barroso et al. (2006), algunos países, como Suiza, ya en el siglo XIX, decidieron desarrollarse como destinos turísticos para la diversificación y desarrollo de sus economías, adoptando estrategias competitivas.
Igualmente, por analogía, se aplica el prinipio de ventaja comparativa de costos (David Ricardo 1817)3, entre dos destinos, cuando uno de estos puede ofrecer a menor costo el servicio turístico.

Posteriormente, Michael E. Porter (1947), con el principio de la ventaja competitiva de las naciones (1991), demostró que las empresas líderes en cualquier campo tienden a agruparse en áreas geográficas relativamente pequeñas denominadas conglomerados - clústers competitivos, altamente eficientes, que permiten crear una ventaja competitiva sostenible. Así, la competitividad se concibe como un fenómeno eminentemente empresarial, pues los productos turísticos se generan en los destinos turísticos por empresas turísticas competitivas, integradas en verdaderos clúster.

El modelo de Porter (1991), conocido como "diamante dinámico", considera cuatro grandes factores que pueden facilitar o impedir las ventajas competitivas de las empresas que operan en una nación determinada, y que son:

1. La dotación del país, en términos de cantidad y calidad de los factores productivos básicos (fuerza de trabajo, recursos naturales, capital e infraestructura), habilidades, conocimientos y tecnologías especializados que determinan su capacidad para generar y asimilar innovaciones.

3 1817, año en que David Ricardo publica Principios de economía política y tributación. 
2. La naturaleza de la demanda interna en relación con la oferta del aparato productivo nacional; en particular, es relevante la presencia de fuertes demandantes que presionan a los oferentes con sus exigencias de artículos innovadores y que se anticipen a sus necesidades.

3. La existencia de una estructura productiva conformada por empresas de distintos tamaños, pero eficientes en escala internacional, relacionadas horizontal y verticalmente, que aliente la competitividad mediante una oferta interna especializada de insumos, tecnologías y habilidades para sustentar un proceso de innovación generalizable a lo largo de cadenas productivas.

4. Las condiciones prevalecientes en el país en materia de creación, organización y manejo de las empresas, así como de competencia, principalmente si está alimentada o inhibida por las regulaciones y las actitudes culturales frente a la innovación, la ganancia y el riesgo.

Además de estos cuatro factores, Porter (1991) agrega asimismo dos variables externas: 1. Los acontecimientos imprevistos (catástrofes medioambientales, atentados terroristas, boicots o embargos políticos, entre otros). 2 . Las influencias de los gobiernos (adopción de políticas económicas, restricciones legales, entre otros). Si bien este modelo está diseñado, en principio, para analizar la competitividad entre naciones, es aplicable también a niveles inferiores de regiones, provincias, ciudades y localidades y destinos turísticos.

Ahora, Crouch y Ritchie (1999) plantean que, además de estos determinantes, hay que tener en cuenta los recursos históricos y culturales, pues los recursos de un destino turístico pueden cambiar a lo largo del tiempo, alterando la ventaja comparativa de un destino turístico.

Así, la base de la competitividad del destino turístico, está determinada por tres clases de recursos: heredados, creados, y recursos complementarios. En el ámbito de la investigación turística, Hassan (2000) asevera que la competitividad de los destinos turísticos es la capacidad de un destino para crear e integrar productos con valor añadido que permitan sostener los recursos locales y conservar su posición de mercado respecto a sus competidores.

En el análisis de la competitividad en los destinos turísticos, Crouch y Ritchie (1999) introducen la teoría de la ventaja comparativa y de la ventaja competitiva. Según estos autores, la ventaja comparativa hace referencia a los factores de los que está dotado el destino turístico, incluyendo tanto a los que ocurren de forma natural como a aquellos que han sido creados.

Sin embargo, Crouch y Ritchie (1999) apuntan que, además de estos cinco grupos (que son válidos para 
cualquier subsector del sector servicios), habría que tomar en cuenta también los recursos históricos y culturales. Las ventajas comparativas de un destino turístico están representadas por el paisaje, la flora, la fauna, el clima y el patrimonio histórico-cultural. Los recursos turísticos, a diferencia de los de una industria, no suelen experimentar agotamiento como resultado de su uso, de allí que la competitividad debe tener un tratamiento diferente.

Según Sánchez et al. (2000), el modelo conceptual de Crouch y Ritchie (1999) no es un modelo predictivo ni causal, sino simplemente explicativo. Para estos autores, la competitividad es la capacidad de un país para crear valor añadido e incrementar, de esta forma, el bienestar nacional mediante la gestión de ventajas y procesos, atractivos, agresividad y proximidad, integrando las relaciones entre los mismos en un modelo económico y social.

Los elementos de este modelo son los siguientes: entorno competitivo (microeconómico), entorno competitivo (macroeconómico), recursos principales y atractivos, exposiciones universales y superestructuras turísticas, factores y recursos complementarios, la gestión del destino y determinantes de calificación.

Garau (2006) piensa que este modelo es una de las primeras aportaciones conceptuales a la competitividad de los destinos turísticos, que explica las razones y los fundamentos del éxito competitivo de los destinos tu- rísticos, pues un destino turístico competitivo es aquel que contribuye, mediante esta actividad, a elevar la calidad de vida de la población residente.

Para Crouch y Ritchie (1999), el turismo es un sistema abierto que está constantemente siendo influido por los continuos cambios del macroentorno, el cual afecta todas las actividades económicas y no solamente las turísticas. Por lo tanto, cualquier evento a nivel mundial puede repercutir en el desarrollo y competitividad de los destinos turísticos. Estos eventos se pueden presentar como oportunidades o amenazas. Estos factores son: económicos, tecnológicos, ecológicos, políticos y legales, socioculturales y demográficos.

A su vez, el microentorno está constituido por las organizaciones turísticas, tanto públicas como privadas, que posibilitan el desarrollo de los destinos turísticos, formando el sistema turístico con el que los destinos deben competir. Este sistema proporciona al turista los elementos básicos para desarrollar su experiencia turística, destacando los recursos turísticos, los hoteles, los restaurantes, los transportes, los comercios, los equipamientos públicos, entre otros, de tal forma que del grado de competitividad y colaboración que exista entre estos va a depender la competitividad del microentorno y, por ende, del destino turístico.

Para Flores (2008), la competitividad es sostenible, si el sistema turístico se conecta a los mercados mediante 
la labor efectuada por los intermediarios, entre los cuales encontramos a los turoperadores, las agencias de viajes, las empresas de viajes de incentivo, entre otros, y aquellas empresas que mejoran el flujo de información, monetario y de conocimiento, tales como entidades financieras, agencias de publicidad, consultoras de mercados, portales de Internet.

El modelo de Ritchie y Crouch se aproxima más a la teoría estructural de la competitividad, pues en él se consideran tanto factores del entorno general -enfoque macroeconómico-, así como aquellos relacionados con los factores microeconómicos y de gestión de las empresas, administraciones públicas y demás organizaciones localizadas en los propios destinos turísticos -enfoque estratégico- y que conforman el sistema turístico.

Entre las principales debilidades de este modelo, puramente descriptivo, se menciona el hecho de que muchos destinos no dispongan de bases de datos para evaluar estos factores descritos; igualmente, se debe hacer una ponderación que indique el orden de importancia de cada uno de estos factores y se debe considerar que no se elaboran indicadores que permitan medir los recursos naturales de los destinos.

\section{Macroentorno: la competitividad del sector turismo en Colombia}

Colombia, desde inicios del siglo XX, dio el primer paso para la identificación del sector turismo como una industria potencial para el crecimiento económico del país con la conformación de la Oficina de Turismo en 1931.

En 1996, se presentó el estudio de competitividad del sector turismo por parte del Ministerio de Comercio Industria y Turismo, basado en el enfoque clásico de Porter, que definió diez grandes clúster: dos vacacionales; costa Caribe y costa Pacífica; cuatro clúster difusos: Boyacá, Eje Cafetero, San Agustín, Tierra Adentro y Ecoturismo (Parques Nacionales Naturales, Llanos Orinoquía y Amazonía) y cuatro clúster a partir de grandes ciudades, Santa fe de Bogotá y zona de influencia, Antioquia y frontera con Panamá, Suroccidente (Cali, Popayán, Pasto) y Santanderes (MINCIT, 2008).

Pero este sector solo tuvo el protagonismo recientemente, en el año 2002, cuando el turismo realmente comenzó a desarrollarse e instaurarse como un motor de crecimiento económico en Colombia, debido a la política de seguridad democrática de los últimos Gobiernos.

Debido a lo anterior y a la recuperación de la confianza por parte de los ciudadanos y del Gobierno, los cuales han jugado un papel importante en todo este proceso, se ha logrado cambiar la apreciación del país 
en el exterior. De otra parte, las ventajas creadas a través de los incentivos tributarios y la recuperación de la inversión extranjera, han sido aspectos claves en la transformación de la industria turística en Colombia.

Según Confecámaras (2013), en su informe sobre el sector turístico en Colombia, con cifras oficiales del Ministerio de Comercio Industria y Turismo, este sector registró un incremento del $4.2 \%$ con respecto a igual periodo de 2012, y el número de viajeros no residentes que ingresaron al país se incrementó en aproximadamente un 10 \%. Igualmente, el Registro Nacional de Turismo tiene inscritas más de 17.000 empresas activas en este campo, incluyendo establecimientos de comercio y personas naturales. Una oferta creciente, compuesta por más de 13.000 compañías, de las cuales, 7.000 son de alojamientos y hospedajes, 4.000 agencias de viajes y más de 800 empresas del subsector gastronómico. Un sector de la economía que contribuye con más de 104.540 empleos y con una gran estabilidad empresarial, pues estas registran un promedio de 27 años (Confecámaras, 2013).

El turismo es un sector que ha mostrado un gran dinamismo, pues el grupo de hotelería y restaurantes ha venido creciendo a un ritmo del $4 \%$ en el 2012, una ocupación hotelera del 53.5 \% , debido a la recuperación de la imagen del país en el extranjero y por ende la mayor seguridad para viajar (MINCIT, 2014).
De hecho, en el año 2012 ingresaron al país 2.429.032 viajeros no residentes en Colombia, un $3.1 \%$ más que en el año 2011. De ellos, 1.591.120 ingresaron al país por los medios aéreos, marítimos y terrestres de control migratorio. 254.351 lo hicieron por cruceros y 583.561 correspondió a colombianos no residentes en el país. Un informe del Ministerio de Comercio industria y Turismo registra que el motivo de viaje de estos turistas es ocio (58,9\%), negocios (31.2\%) y participación en convenciones (6.2\%) (MINCIT, 2014).

La llegada de pasajeros por vía aérea se incrementó en un $21 \%$ con respecto al año 2012, hasta 1.541.080, siendo el aeropuerto de Río Negro el que más registró con 84.5 \%, luego Cartagena con el 56.4 \% y Barranquilla con el 31.2 \% y con el transporte terrestre se movilizaron 109.721.370 pasajeros (MINCIT, 2014).

Los parques nacionales registraron un $18.9 \%$ más de visitantes que el año 2011, al pasar de 694.148 visitantes en el año 20011 a 825.497 visitantes en el año 2012, concentrándose en la región del Caribe con el $88.6 \%$, luego la región andina con el 10,5 \%, el Pacífico el 0,6\%, Amazonía y Orinoquia con el $0.3 \%$. Los parques que registraron mayor crecimiento: El Cocuy con el $49.2 \%$ y Chingaza $(45,5 \%)$ e Iguaque (44.9\%) (MINCIT, 2014).

Sin embargo, el Foro Económico Mundial (FEM) publicó el Reporte de Competitividad de Viajes y Turismo 
(RCVT) y los resultados obtenidos por Colombia en el RCVT-2013, así como un vistazo a la situación global y latinoamericana. La calificación obtenida por Colombia en el ICVT disminuyó de 3,94 en 2011 a 3,90 en 2013. El menor puntaje $(-0,04)$ registrado hace que este año se dé un retroceso de 7 puestos con respecto al resultado de 2011, año en el que Colombia se había ubicado en el puesto 77. De igual forma, en 2013 Colombia disminuyó su posición relativa (porcentaje de países superados por Colombia), la cual pasó de 44,6 \% en 2011 a 40 \% (DNP, 2013).

Por subíndices, Colombia mantuvo casi estable su posición en marco regulatorio (ascendió un puesto), subió cinco posiciones en recursos humanos, culturales y naturales, mientras que descendió once casillas en el subíndice de ambiente de negocios e infraestructura. Es decir, que es a aquella última categoría a la cual se debe en mayor medida el descenso global de Colombia, en cuanto cuatro de los cinco pilares que componen este subíndice presentaron caídas superiores a 10 posiciones. Con todo, la mayor caída en un pilar se da en el tema de sostenibilidad ambiental (bajó 20 casillas), perteneciente a la categoría marco regulatorio (DNP, 2013).

A nivel global, Suiza se mantiene como el país más competitivo, a la vez que las primeras posiciones siguen ocupadas en buena medida por países europeos. En América Latina, Colombia se sostiene en la casilla 11, mientras que Panamá se convirtió en el país más competitivo, desplazando a México que había sido catalogado líder regional en 2009 y 2011 (DNP, 2013).

Para el año 2014, la situación ha evolucionado contradiciendo el indicador de competitividad presentado para el año 2013 y que evidencia que este indicador puede mejorar.

A nivel regional, sobre el tema de la competitividad en turismo, se registran como antecedentes de política pública el reconocimiento de una serie de debilidades en cuanto a competitividad, las cuales se han pretendido fortalecer mediante la firma de convenios de competitividad.

Para el caso de Boyacá, se firmó el Convenio de Competitividad Turística Departamento de Boyacá, en el año 2009. En este convenio, que busca una planeación concertada entre el sector público y privado, se fijó como visión: "Para el año 2025 Boyacá estará dentro de los diez principales destinos ecoturísticos y culturales reconocidos a nivel latinoamericano (DNP, 2009).

Se parte de los siguientes aspectos que identifican el problema de la baja competitividad del sector en los siguientes aspectos:

1. Inexistencia de una política pública de turismo en el departamento. 
2. Débil planificación turística en el departamento de Boyacá.

3. Débil diseño del producto turístico.

4. Deficiencias en la infraestructura básica.

Igualmente, la Agenda 2032 (Comisión Regional Competitividad, 2008) define la siguiente visión: Boyacá en el año 2032 será una región articulada con el desarrollo mundial y altamente competitiva; con un desarrollo económico sostenible, sustentable e inclusivo, que le permita tener un crecimiento sostenido del $6 \%$ anual en el PIB, mediante la oferta competitiva de bienes y servicios relacionados con el turismo, la agroindustria, la minería, el medio ambiente y el conocimiento, soportada en una sociedad autogestionaria líder de los procesos regionales, un tejido empresarial consolidado y un capital institucional que corresponda a la dinámica económica. Con esto se evidencia que el turismo es considerado un sector estratégico para el desarrollo de Boyacá.

Ante estas proyecciones, el Gobierno departamental y local ha asumido el reto de ejecutar estas políticas con proyectos como desarrollar el portafolio de productos turísticos del departamento de Boyacá.

Bajo el argumento de que Boyacá es un departamento con enormes potencialidades, por las bondades que ofrecen sus paisajes, sus recursos naturales, sus gentes y los servicios que se ofrecen, como el turismo, el Gobierno de Boyacá lidera las acciones de carácter político y administrativo orientadas a consolidar en el imaginario colectivo, nacional e internacional, el concepto de que Boyacá es una maravilla.

En razón a las múltiples riquezas de esta región del país, fue necesario determinar siete grandes atractivos para orientar una estrategia de turismo y competitividad en torno a lugares como la Sierra Nevada del Cocuy, el Lago de Tota, Tunja, el piedemonte llanero, el occidente, Villa de Leyva y Paipa.

Cuando se ha pensado que el turismo es una de las apuestas productivas de una región como Boyacá con sus potencialidades de desarrollo, es porque al ser Colombia uno de los países en vías desarrollo, se ha considerado que la competitividad puede permitir a las empresas sobreponerse a las limitaciones de sus reducidos mercados internos para poder incursionar con éxito en los mercados internacionales.

Por eso, Villa de Leyva, municipio con gran vocación turística por sus atractivos naturales, culturales e históricos, vive las consecuencias de un acelerado cambio en su vocación, de pasar de una economía rural con un pequeño centro histórico, se ha convertido en uno de los referentes de la industria turística del país con un acervo patrimonial diverso. 


\section{Metodología}

La técnica Delphi es un medio efectivo para construir consenso en un grupo, sin que los integrantes se reúnan físicamente. Desde su elaboración, hace más de 50 años, la técnica Delphi ha gana ganado amplia popularidad a través de las disciplinas científicas como técnica de investigación (Sancho, s.f.).

El método Delphi se concibe como una técnica de investigación cualitativa, alternativa a las convencionales, la cual según Turoff, quien fue uno de los pioneros en su utilización, mediante un estudio de experiencias de empleo del Delphi en turismo (1971), se puede definir como "un método de estructurar un proceso de comunicación grupal para que el proceso sea efectivo, al permitir tratar a un grupo de individuos como un conjunto para solucionar un problema complejo" (Betancort \& Fernández, 2002).Debido a la complejidad del objeto de estudio se consideró apropiado el uso de esta metodología Delphi, pues el conocimiento de expertos del sector nos permite mayor objetividad sobre la competitividad de Villa de Leyva.

Para el caso, se analizó cada uno de los parámetros definidos por Porter, conocido como las " 5 fuerzas de Porter",que intenta determinar las presiones que ejercen los distintos elementos dentro de un sector $y$, en definitiva, evaluar el grado de atractivo del mismo, lo que se traduce en potencial de competitividad.
Este modelo clásico de Porter nos muestra la situación competitiva de un sector, turístico en este caso, en función de cinco grupos de variables, de cuyos movimientos o características dependerán los resultados de las empresas instaladas en dicho sector al optar por estrategias adaptadas al estado de dichas variables.

Evaluación de expertos: no todos los parámetros y factores se pueden considerar igual de importantes, por lo tanto con el grupo de expertos se hace una ponderación con el fin de darle un peso en términos porcentuales a cada factor.

Aplicación empírica. Finalmente para considerar la evaluación de los componentes de la competitividad y su grado de importancia, se evaluó con la calificación que dieron los expertos a cada una de las variables, con el fin de poder concluir cuál es la condición competitiva de Villa de Leyva como destino internacional.

Por tanto, tal como indica el procedimiento metodológico de Delphi, se aplicó una encuesta a expertos, para que dieran su opinión sobre el nivel de importancia de cada una de las 30 variables de los cuatro factores del Diamante de Porter en el nivel de competitividad de un destino turístico internacional, calificando cada parámetro con una escala de valoración cualitativa, siendo, "nada importante", "medianamente importante", "regularmente importante", "importante" y "muy importante", que, equiparada en términos numé- 
ricos, corresponde a una escala desde 1.0 a 5.0, siendo este último "muy importante".

\section{Resultados}

La primera columna de la Tabla 1 presenta el promedio en términos numéricos de la calificación dada por los expertos, calificación que se presenta en la segunda columna del cuadro 1, expresada en porcentaje. Este porcentaje se obtuvo considerando que la nota de 5.0 es igual al $100 \%$. Una calificación menor a 5.0 será menor al $100 \%$.

En cuanto al análisis empírico, se aplicó una segunda encuesta a los expertos para calificar el estado actual de la competitividad de Villa de Leyva como destino turístico internacional, durante los meses de marzo a abril del 2014. La escala de Likert empleada (de 1.0 al 5.0 en números), desde "muy malo", "malo", "regular", "bueno" y "excelente".

Industrias de soporte y relacionadas. Como puede observarse en la Tabla 1, los expertos participantes en el Delphi consideraron que una de las condiciones de competitividad de un destino cultural más importantes es la conservación (4.50), siempre basándose en una escala de Likert "nada importante", "medianamente importante", "regularmente importante", "importante" y "muy importante", que es un concepto que incluye el patrimonio histórico y cultural, incluso la conserva- ción del medio ambiente, la fauna y flora. Le siguen en importancia: proveedores de infraestructura $(4,26)$, servicios de hospitalidad $(4,35)$.

Esta calificación promedio ponderado dada por los expertos se presenta en la tercera columna de la Tabla 1. La calificación definitiva de cada una de las variables determinantes de la competitividad de Villa de Leyva es el resultado de multiplicar la calificación promedia dada por los expertos sobre la percepción y conocimiento que tienen de cada variable por el peso dada a las mismas en términos porcentuales.

Ahora, en la calificación ponderada de estas variables para Villa de Leyva, de acuerdo con la opinión de los expertos, manteniendo la escala de Likert que va desde "muy malo", "malo", "regular" "bueno" y "excelente", son considerados "regular", la conservación (3.60), los servicios de hospitalidad (3,39), los servicios de seguridad (3.27) y el promedio ponderado del factor calificado con 3.12 .

Condiciones de los factores. Según los expertos que participaron en el estudio, las condiciones de los factores más importantes que hacen que un destino histórico cultural sea competitivo son: recursos naturales, recursos culturales y atracciones $(4,60)$, recursos humanos $(4,50)$, atracciones hechas por el hombre y otras actividades $(4,4)$, y niveles de los servicios $(4,50)$. 
Una evaluación de estos recursos para Villa de Leyva que corresponde a la cualidad de "bueno" corresponde a recursos naturales, recursos culturales y atracciones con (4.14), recursos humanos $(4,05)$. Las demás condiciones de competitividad son consideradas "regular", pues su calificación ponderada está en el intervalo de 3.0 a 4.0.

Estructura de la industria y rivalidad. Según los expertos, las condiciones de la estructura de la industria más importantes que hacen que un destino histórico cultural sea competitivo son: competitividad de precios $(4,50)$, le siguen los estándares de calidad $(4,30)$, la madurez competitiva (4.15).

Condiciones de la demanda. Para los expertos, esta variable fue considera como muy importante con (4.60), le sigue en importancia la expansión y segmentación del mercado. Esto quiere decir que los destinos en el contexto internacional ofrecen productos turísticos diseñados para segmentos especiales
Tabla 1. Matriz de evaluación de competitividad de Villa de Leyva como destino turístico

\begin{tabular}{|c|c|c|c|c|}
\hline $\begin{array}{c}\text { Industrias de soporte } \\
\text { y relacionadas }\end{array}$ & Importancia & Peso \% & Calificación & $\begin{array}{c}\text { Condición } \\
\text { compet. }\end{array}$ \\
\hline Servicios de hospitalidad & 4,35 & 87,00 & 3,90 & 3,39 \\
\hline Servicios de viaje & 3,49 & 69,80 & 3,50 & 2,44 \\
\hline Proveedores de infraestructura & 4,26 & 85,20 & 3,60 & 3,07 \\
\hline Servicios de transporte público & 3,65 & 73,00 & 3,70 & 2,70 \\
\hline Conservación & 4,50 & 90,00 & 4,00 & 3,60 \\
\hline Servicios de seguridad & 3,80 & 76,00 & 4,30 & 3,27 \\
\hline Limpieza, estética & 4,15 & 83,00 & 4,00 & 3,32 \\
\hline Otros & 4,00 & 80,00 & 4,00 & 3,20 \\
\hline $\begin{array}{l}\text { Condiciones de } \\
\text { los factores }\end{array}$ & Importancia & Peso \% & Calificación & $\begin{array}{l}\text { Condición } \\
\text { compet. }\end{array}$ \\
\hline $\begin{array}{c}\text { Recursos naturales, recursos } \\
\text { culturales y atracciones }\end{array}$ & 4,60 & 92,00 & 4,50 & 4,14 \\
\hline $\begin{array}{l}\text { Atracciones hechas por el } \\
\text { hombre y otras actividades }\end{array}$ & 4,45 & 89,00 & 4,00 & 3,56 \\
\hline Recursos humanos & 4,50 & 90,00 & 4,50 & 4,05 \\
\hline Recursos intangibles & 4,00 & 80,00 & 4,50 & 3,60 \\
\hline $\begin{array}{c}\text { Percepción e } \\
\text { identificación de la marca }\end{array}$ & 4,00 & 80,00 & 3,00 & 2,40 \\
\hline $\begin{array}{l}\text { Percepciones sobre } \\
\text { seguridad y sanidad }\end{array}$ & 3,80 & 76,00 & 4,00 & 3,04 \\
\hline Niveles de los servicios & 4,50 & 90,00 & 4,00 & 3,60 \\
\hline Distancia de los mercados & 4,20 & 84,00 & 4,00 & 3,36 \\
\hline Otros & 4,00 & 80,00 & 4,00 & 3,20 \\
\hline
\end{tabular}




\begin{tabular}{|c|c|c|c|c|}
\hline $\begin{array}{l}\text { Estructura de la industria, } \\
\text { estrategia y rivalidad }\end{array}$ & Importancia & Peso \% & Calificación & $\begin{array}{l}\text { Condición } \\
\text { compet. }\end{array}$ \\
\hline Dificultades institucionales & 3,65 & 73,00 & 3,50 & 2,60 \\
\hline Competitividad de precios & 4,50 & 90,00 & 3,50 & 3,15 \\
\hline Diversidad de instalaciones y servicios & 4,00 & 80,00 & 4,00 & 3,20 \\
\hline Estándares de calidad & 4,30 & 86,00 & 4,50 & 3,87 \\
\hline Madurez competitiva & 4,15 & 83,00 & 4,00 & 3,32 \\
\hline Sagacidad tecnológica & 3,85 & 77,00 & 3,00 & 2,31 \\
\hline $\begin{array}{l}\text { Condiciones de } \\
\text { la demanda }\end{array}$ & Importancia & Peso \% & Calificación & $\begin{array}{c}\text { Condición } \\
\text { compet. }\end{array}$ \\
\hline Tendencias y patrones de crecimiento & 4,60 & 92,00 & 4,6 & 4,23 \\
\hline Sensibilidad a los precios & 3,71 & 74,20 & 4,3 & 3,19 \\
\hline Preferencias y tendencias del mercado & 3,45 & 69,00 & 4,5 & 3,11 \\
\hline $\begin{array}{c}\text { Expansión de la segmentación } \\
\text { del mercado }\end{array}$ & 4,50 & 90,00 & 4,3 & 3,87 \\
\hline $\begin{array}{l}\text { Condiciones de crecimiento } \\
\text { económico en mercados objeto }\end{array}$ & 3,58 & 71,60 & 4,2 & 3,01 \\
\hline Canales de venta y reservas & 3,35 & 67,00 & 4,2 & 2,81 \\
\hline
\end{tabular}

Fuente: encuestas presente estudio, junio de 2014.

del mercado, es decir, atendiendo las necesidades del consumidor, sus expectativas y requerimientos.

Los expertos consideraron que Villa de Leyva tiene un potencial de desarrollo turístico "bueno" en general, sin embargo, como la evaluación fue ponderada, la calificación del factor condiciones de la demanda fue calificado como "regular", es decir, con un valor de 3,37.

La gestión pública turística. Otro aspecto considerado por Porter es la participación del Gobierno en el mejoramiento de las condiciones competitivas. Es evidente que el turismo en Villa de Leyva ocupa uno de los primeros renglones de la economía, sin embargo, esta actividad no cuenta con la planificación y control necesarios para lograr un desarrollo sostenible. Por el contrario, se han venido desarrollando fenómenos opuestos al concepto de competitividad y sostenibilidad, ya que ni la población, ni la administración pública local, ni los prestadores de servicios, son conscientes de la complejidad de esta actividad, presentándose de esta forma un movimiento hotelero bastante significativo y una demanda incontrolada y depredadora, que surge principalmente de los eventos programados, como lo son el "Festival de Cometas" y el "Festival de Luces", los cuales generan más impactos negativos que positivos en los aspectos económicos, sociales y culturales. Según los resultados de un seguimiento realizado al número de visitantes en el Festival de las Cometas, el municipio llegó a recibir hasta 40.000 personas en un solo día. 
La demanda estaba basada en el producto turístico cultural, siendo este segmento selectivo, poco repetitivo y que solo se renueva entre capas de la población relativamente altas, se empezó a dar una pérdida de demanda, provocando la búsqueda de mercado alternativos que por no ser organizados produjeron un efecto negativo sobre los atractivos.

Los impactos negativos de orden ambiental y social causados por esta demanda turística deben estimarse como posibles amenazas para el desarrollo del turismo en la zona y por esto es necesario que se manejen conceptos de capacidad de carga no solo en el centro histórico y los principales atractivos culturales de la zona urbana, sino también en todos los atractivos naturales y el "Santuario de Flora y Fauna" para una mejor orientación de la vida del territorio.

En el Plan de Ordenamiento Territorial de Villa de Leyva (2004) se recomiendan estrategias en búsqueda del desarrollo turístico sostenible, donde el municipio debe ofrecer un producto turístico centrado en la cultura y en las actividades recreativas y deportivas, con un alto sentido ambientalista, de tal manera que se conforme en su entorno urbano y rural un ambiente creativo y dinámico alrededor de estos dos atractivos.

En conclusión, el concepto de competitividad, tanto si se aborda desde una óptica macroeconómica como si se hace desde una microeconómica, es un concepto lo suficientemente complejo y amplio como para que las diferentes instituciones y los investigadores de la materia no hayan consensuado una única, y generalmente aceptada, definición de competitividad.

Para considerar la competitividad de un destino turístico como Villa de Leyva, esta no solo deber tener ventajas comparativas, sino que precisa además de ventajas competitivas, es decir, no solo es necesario poseer una variedad, más o menos amplia, de productos y recursos turísticos, sino que estos deben ser gestionados de forma eficiente a medio y largo plazo.

Villa de Leyva tiene un gran potencial turístico, pero no se ha consolidado como un buen destino, pues según la percepción de expertos, los determinantes de la competitividad en su mayoría son considerados "regulares" dentro de una escala de Likert, de forma tal, que todo está por hacer, mejorar y reestructurar, como la infraestructura turística, la capacitación en la prestación de servicios, la adecuada asignación de recursos gubernamentales y privados para el sector, en aquellos determinantes claves de éxito, los cuales son definitivos para poder construir un verdadero destino turístico competitivo y especialmente la falta de un plan de mercadeo estratégico que permita posicionar a Villa de Leyva como destino turístico internacional.

Villa de Leyva debe ser posicionado en la mente de los turistas por ser uno de los mejores conjuntos arquitec- 
tónicos contextuales como patrón urbano cultural del mundo, lo cual, a pesar de las informalidades y falencias de su estructura turística, representa el principal insumo para poder tener visión de futuro frente al desarrollo sostenible del municipio y sus habitantes.

Por esto, todo aquello relacionado con la oferta y la demanda del turismo debe forjase basado en unos parámetros bien establecidos en donde se planifique de la mejor manera el destino para beneficios de la tierra y sus pobladores.

Cualquier esfuerzo para conseguir un destino más competitivo a los ojos del país y del mundo, debe basarse en un reconocimiento propio y en una interacción total de fuerzas en donde la comunidad anfitriona, sin lugar a dudas, debe ser líder en todo el proceso. De no existir esta integración, se seguirá en el mismo círculo vicioso actual en donde no existe retroalimentación alguna que pueda generar beneficios para el municipio, sino, en cambio, un desmembramiento del tejido social, económico y cultural del territorio, lo que va en contra del concepto de competitividad.

\section{Conclusiones}

Para considerar la competitividad de un destino turístico como Villa de Leyva, esta no solo deber tener ventajas comparativas, sino que precisa además de ventajas competitivas, es decir, no solo es necesario poseer una variedad, más o menos amplia, de productos y recursos turísticos, sino que estos deben ser gestionados de forma eficiente a medio y largo plazo.

Villa de Leyva tiene un gran potencial turístico, pero no se ha consolidado como destino competitivo, pues según la percepción de expertos, los determinantes de la competitividad en su mayoría son considerados "regulares" dentro de una escala de Likert, de manera tal, que todo está por hacer, mejorar y reestructurar, como la infraestructura turística, la capacitación en la prestación de servicios, la adecuada asignación de recursos gubernamentales y privados para el sector, en aquellos determinantes claves de éxito, los cuales son definitivos para poder construir un verdadero destino turístico competitivo. Con relación a las industrias de soporte y relacionadas, la conservación es la variable con mayor calificación $(3,60)$, luego le siguen los servicios de hospitalidad (3.39); deben mejorar los servicios de viaje $(2,44)$ y los servicios de transporte público $(2,70)$, estos últimos calificados como deficientes en términos competitivos.

Las condiciones de los factores, los recursos naturales, recursos culturales y atracciones se destacan con una buena calificación (4.14) al igual que los recursos humanos $(4,05)$, pero no se percibe una identidad o una marca (2.40), lo que implica una trabajo concertado de 
todos los actores del sistema turístico para conseguir un destino más competitivo a los ojos del país y del mundo, el cual debe basarse en un reconocimiento propio y en una interacción total de fuerzas en donde la comunidad anfitriona sin lugar a dudas debe ser líder en todo el proceso, pues las condiciones de la deman$\mathrm{da}$, las tendencias y patrones de crecimiento fueron evaluadas con 4,23.

Sin embargo, las variables de estructura, estrategia y rivalidad, evaluadas con valores que en promedio no sobrepasan de 3.0, nos muestran un destino que debe integrar más su oferta de servicios, para hacer que el visitante reciba un mayor valor agregado y su percepción en cuanto a competitividad sea mejor evaluada.

De no existir esta integración se seguirá en el mismo círculo vicioso actual en donde no existe retroalimentación alguna que pueda generar beneficios para el municipio, sino, al contrario, un desmembramiento del tejido social, económico y cultural del territorio. 


\section{Referencias}

Alcaldía de Villa de Leyva. (2004). Plan de ordenamiento territorial -POT-. Villa de Leyva: Alcaldía.

Barroso, M. O. et al. (2006). La competitividad internacional de los destinos turísticos: del enfoque macroeconómico al enfoque estratégico. Cuadernos de Turismo, 17, 24.

Betancort, B. \& Fernández, H. (2002). Determinantes de la competitividad de un destino turístico: los casos de Puerto del Carmen, Costa Teguise y Playa Blanca. Recuperado de http://www.datosdelanzarote.com/ uploads/doc/20051216194706294deterdemanda.pdf

Comisión Regional de Competitividad Boyacá 2008-2032. (2008). Competitividad. Recuperado de: http://competitividad.com.co/Boyaca/PRC

Confecámaras. (2013) Crece el sector turismo en Colombia. Retos del sector turismo. Recuperado de http://www.confecamaras.org.co/noticias/237-crece-el-sector-del-turismo-en-colombia

Crouch, G. I. \& Ritchie, J. R. B. (1999). Tourism, competitiveness and societal prosperity. Journal of Business Research, 44 (3), 137-152.

Flores, D. (2008). Competitividad sostenible de los espacios naturales protegidos como destinos turísticos: un análisis comparativo de los parques naturales Sierra de Aracena y Picos de Aroche y Sierras de Cazorla, Segura y Las Villas. Huelva: Universidad de Huelva. 
Garau, J. (2006). Propuesta de dos indicadores para la mediación de la competitividad de los destinos de sol y playa del mediterráneo: avance de resultados desde el punto de vista de la demanda. En XV Simposio Internacional de Turismo y Ocio, llevado a cabo en Barcelona, ESADE, Fira.

Hassan, S.S. (2000). Determinants of market competitiveness in an environmentally sustainable tourism industry. Journal of Travel Research, 38, 239-245.

Ivanchevich, J., Lorenzi, P., Skinner, S. \& Crosby, P. (1996). Gestión: calidad y competitividad. Madrid: Universidad del Cauca.

Ministerio de Comercio Industria y Turismo -MINCIT-. (2008). Asistencia técnica en planificación del turismo. Bogotá: MINCIT, Dirección de calidad y desarrollo sostenible, Grupo de Planificación y Desarrollo Sostenible.

Ministerio de Comercio Industria y Turismo -MINCIT-. (2008). Manual para la destinación de los recursos y presentación de proyectos. Bogotá: MINCIT, Fondo de Promoción Turística, Viceministerio de Turismo.

Ministerio de Comercio Industria y Turismo -MINCIT-. (2008). Sistema de indicadores de competitividad para el turismo colombiano. Bogotá: MINCIT, Viceministerio de Turismo.

Ministerio de Comercio Industria y Turismo -MINCIT-. (2014, febrero). Informe de turismo. Bogotá: Oficina de Estudios Económicos. 
Ministerio de Comercio Industria y Turismo -MINCIT-. (2014). Plan sectorial de turismo 2014-2018. Bogotá: MINCIT, Viceministerio de Turismo.

Porter, M.E. (1991). La ventaja competitiva de las naciones. Buenos Aires, Argentina: Vergara.

Ritchie, J.R.B. \& Crouch, G.I. (2000). The competitive destination. A sustainable perspective. Tourism Management, 21, 1-7.

Sánchez, M. \& Fajardo, M. (2000). La competitividad de los destinos turísticos: un análisis cuantitativo mediante modelos logísticos. Aplicación a los municipios extremeños. Recuperado de http://150.214.182.12:8080/ turismo/turismonet1/economia\%20del\%20turismo/mercados\%20turisticos/competitividad\%20de\%20los\%20destinos\%20turisticos.pdf

Sancho, A. (s.f.). Apuntes de metodología de la investigación en turismo. Recuperado de http://snap3.uas.mx/RECURSO1/libroselectronicos/turismo/Apuntes_de_metodologia_de_la_investigacion_en_turismo.pdf 